\title{
On a Curvature Property of Effective Divisors and Its Application to Sheaf Cohomology
}

\author{
By \\ Hiroshi FusE* and Takeo OHSAWA**
}

\begin{abstract}
Exploring a method of taming the boundary behavior of $n$-convex exhaustion functions, a curvature property of line bundles associated to effective Cartier divisors is proved. Cohomology vanishing theorems of the Serre type and the Kodaira-Nakano type are obtained as application.
\end{abstract}

\section{Introduction}

Let $X$ be a complex analytic space of dimension $n$. It is known that $X$ is $n$-complete in the sense of Andreotti-Grauert [A-G] (see section one for the definition) if every irreducible component of $X$ is noncompact (cf. [O-3], $[\mathrm{Dm}])$. This shows that the vanishing theorem for the cohomology groups of top degrees, due to Y.-T. Siu [S], is essentially contained in [A-G].

In $[\mathrm{F}]$, based on the 1-completeness of noncompact Riemann surfaces, an elementary proof was given to a basic fact that, for any Riemann surface $R$ and for any point $p \in R$, the line bundle $[p]$ associated to the divisor $p$, is positive.

The purpose of the present note is to extend the paper $[\mathrm{F}]$ to establish the following.

Theorem 1. Let $X$ be a compact complex analytic space of dimension $n$ and let $D$ be an effective Cartier divisor of $X$ such that $|D|$, the support of

\footnotetext{
Communicated by M. Kashiwara. Received March 30, 2009. Revised April 22, 2009.

2000 Mathematics Subject Classification(s): Primary 32A36; Secondary 14J60.

${ }^{*}$ Graduate School of Mathematics, Nagoya University, 464-8602 Chikusaku, Nagoya, Japan.

e-mail: my_hobby_is_math@hotmail.com

${ }^{* *}$ Graduate School of Mathematics, Nagoya University, 464-8602 Chikusaku, Nagoya,

Japan.

e-mail: ohsawa@math.nagoya-u.ac.jp
}

(c) 2009 Research Institute for Mathematical Sciences, Kyoto University. All rights reserved. 
$D$, intersects every $n$-dimensional irreducible component of $X$. Then the line bundle $[D]$ is n-concave (see section one for the definition).

Theorem 1 supplements [O-3] and [Dm]. By applying it we shall show at first the following Serre type vanishing theorem.

Theorem 2. Let $M$ be a complex manifold, let $Z$ be a complex analytic space, let $f: M \longrightarrow Z$ be a proper holomorphic map, let $D$ be an effective divisor of $M$, let $z \in f(|D|)$, and let $n$ be any positive integer exceeding the dimension of any compact irreducible component of $\left(f^{-1}(z) \backslash|D|\right) \cup\left(f^{-1}(z) \cap|D|\right)$. Then, for any holomorphic vector bundle $E \longrightarrow M$, there exists a positive number $m_{0}$ such that

$$
\left(R^{n} f_{*} \mathcal{O}\left(E \otimes[D]^{m}\right)_{z}=0\right.
$$

holds if $m \geq m_{0}$. Here $\mathcal{O}\left(E \otimes[D]^{m}\right)$ denotes the sheaf of the germs of holomorphic sections of $E \otimes[D]^{m}$, and $R^{n} f_{*} \mathcal{O}\left(E \otimes[D]^{m}\right)$ the n-th direct image of $\mathcal{O}\left(E \otimes[D]^{m}\right)$ by $f$.

For the proof of Theorem 2, we need results from [B] and [O-3].

Further, by employing a method in [O-2], we obtain a refined version of Theorem 2 when $E$ is the canonical bundle of $M$.

Theorem 3. In the above situation, suppose moreover that $E$ is the canonical bundle $K_{M}$ of $M$, and that $M$ admits a Kähler metric. Then

$$
\left(R^{n} f_{*} \mathcal{O}\left(K_{M} \otimes[D]\right)\right)_{z}=0
$$

holds.

Theorem 3 may well be regarded as a supplement to Theorem 3.1 and Theorem 4.5 in [O-2], which are extensions of the Kodaira-Nakano vanishing.

\section{$\S 1 . \quad$ q-Complete Spaces and q-Concave Bundles}

Let $X$ be a complex analytic space of dimension $n$. A real valued $C^{2}$ function $\varphi$ defined on an open set $U \subset X$ is said to be $q$-convex if, for any point $x \in U$, there exist a neighborhood $V \ni x$, a holomorphic embedding $\iota$ of $V$ into a domain $\Omega$ of $\mathbb{C}^{N}$ for some $N \in \mathbb{N}$, and a real valued $C^{2}$ function $\Phi$ on $\Omega$ such that $\iota^{*} \Phi=\varphi \mid V$ and that the complex Hessian $\partial \bar{\partial} \Phi$ has everywhere at least $N-q+1$ positive eigenvalues on $\Omega$.

Definition 1. $X$ is called a $q$-complete space (in the sense of $[\mathrm{A}-\mathrm{G}]$ ), if there exists a $q$-convex exhaustion function on $X$. 
Here, an exhaustion function on a topological space is by definition a real valued function whose sublevel sets are all relatively compact.

Let $L \longrightarrow X$ be a holomorphic line bundle and let $\left\{e_{\alpha \beta}\right\}_{\alpha, \beta \in \Lambda}$ be a system of transition functions of $L$ associated to an open covering $\left\{U_{\alpha}\right\}_{\alpha \in \Lambda}$ such that $\pi^{-1}\left(U_{\alpha}\right)$ is equivalent to the product $U_{\alpha} \times \mathbb{C}$. Then a fiber metric of $L$ is naturally identified with a system of positive $C^{\infty}$ functions $h=\left\{h_{\alpha}\right\}, h_{\alpha}$ being defined on $U_{\alpha}$, such that $h_{\alpha}=\left|e_{\alpha \beta}\right|^{2} h_{\beta}$ holds on $U_{\alpha} \cap U_{\beta}$. The pair $(L, h)$ is called a Hermitian line bundle over $X$.

Definition 2. A Hermitian line bundle $(L, h)$ is said to be $q$-concave if the functions $-\log h_{\alpha}$ are all $q$-convex.

\section{$\S 2 . \quad$ Proof of Theorem 1}

Let $X$ be a compact complex analytic space and let $\operatorname{Sing} X$ be the set of singular points of $X$, with respect to the reduced structure. We put

$$
X_{k}=\underbrace{\operatorname{Sing}(\operatorname{Sing}(\cdots \operatorname{Sing} X) \cdots)}_{k}
$$

and $X_{0}=X$. Let $X_{k}^{j}$ be the union of $j$-dimensional irreducible components of $X_{k}$, and let $X^{j}=\cup_{k} X_{k}^{j}$.

Let $D$ be an effective Cartier divisor of $X$, let $s=\left\{s_{\alpha}\right\}$ be a system of local defining functions of $D, s_{\alpha}$ being defined on an open set $U_{\alpha} \subset X$, and let $\left\{e_{\alpha \beta}\right\}$ be the system of transition functions of $[D]$ such that $s_{\alpha}=e_{\alpha \beta} s_{\beta}$ holds on $U_{\alpha} \cap U_{\beta}$, for every $\alpha$ and $\beta$.

A fiber metric of $[D]$ is then a system of positive $C^{\infty}$ functions $h=\left\{h_{\alpha}\right\}$, $h_{\alpha}$ defined on $U_{\alpha}$, such that $h_{\alpha}\left|s_{\alpha}\right|^{2}=h_{\beta}\left|s_{\beta}\right|^{2}$ holds on $U_{\alpha} \cap U_{\beta}$. We fix a fiber metric $h$ of $[D]$ and denote by $|s|^{2}$ the function on $X$ defined by $h_{\alpha}\left|s_{\alpha}\right|^{2}$ on each $U_{\alpha}$.

In order to find a metric $\tilde{h}=\left\{\tilde{h}_{\alpha}\right\}$ of $[D]$ such that $-\log \tilde{h}_{\alpha}$ are $n$-convex, we shall at first find a $C^{\infty}$ function $\eta$ on $X$ such that $-\log |s|^{2}+\eta$ is $n$-convex outside some compact subset of $X \backslash|D|$. After that, we shall modify $-\log |s|^{2}+\eta$ on a compact set, to obtain an $n$-convex exhaustion function $\Phi$ on $X \backslash|D|$ and put $\tilde{h}_{\alpha}=e^{-\Phi}\left|s_{\alpha}\right|^{-2}$. For this purpose, the following is crucial.

Lemma 1. Let $Y$ be a complex analytic space of dimension $n$ equipped with an n-convex exhaustion function $\varphi$, and let $\psi$ be a $C^{\infty}$ exhaustion function on $Y$ such that $\psi$ is n-convex outside a compact subset $K$ of $Y$. Suppose that $\varphi \mid\left(Y^{j} \backslash K\right)$ and $\psi \mid\left(Y^{j} \backslash K\right)$ do not have local maximums for $j>0$. Then there 
exist a compact set $\hat{K} \subset Y$, a positive number $\varepsilon$, a real number $C$, and a $C^{\infty}$ n-convex function $\Phi$ on $Y$ such that $\Phi \mid K=\varepsilon \varphi$ and $\Phi \mid(Y \backslash \hat{K})=\psi-C$.

Proof. Take any $c_{1} \in \mathbb{R}$ such that $\varphi \mid K<c_{1}$ and that $\psi \mid \varphi^{-1}\left(\left(c_{1}, \infty\right)\right)$ is $n$-convex.Then, take $c_{2}$ and $c_{3}$ in such a way that $\psi \mid \varphi^{-1}\left(\left(-\infty, c_{1}\right]\right)<c_{2}$ and $\varphi \mid \psi^{-1}\left(\left(-\infty, c_{2}\right]\right)<c_{3}$.

Then we put, for any $A>0$,

$$
\varphi_{A}=\left\{\begin{array}{lll}
A\left(\psi-c_{2}\right) & \text { on } & \varphi^{-1}\left(\left[c_{3}, \infty\right)\right) \\
\max \left\{\varphi, A\left(\psi-c_{2}\right)\right\} & \text { on } & \varphi^{-1}\left(\left(c_{1}, c_{3}\right)\right) \\
\varphi & \text { on } & \varphi^{-1}\left(\left(-\infty, c_{1}\right]\right)
\end{array}\right.
$$

Although $\varphi_{A}$ is not n-convex in general, it is clearly continuous for sufficiently large $A$, and has no local maximum because so do $\varphi$ and $\psi \mid \varphi^{-1}\left(\left(c_{1}, \infty\right)\right)$. Note that $\varphi_{A} \mid\left(Y^{j} \backslash K\right)$ has no local maximum if $j>0$ by assumption.

After fixing such a number $A$, take a $C^{\infty}$ function $\tilde{\varphi}$ by approximating $\varphi_{A}$, such that $\tilde{\varphi}=\varphi_{A}$ on $Y \backslash \varphi^{-1}\left(\left(c_{1}, c_{3}\right)\right)$ and $\tilde{\varphi} \mid\left(Y^{j} \backslash K\right)$ have no local maximum for $j>0$. We may assume that the critical points of $\tilde{\varphi} \mid Y^{j} \cap \varphi^{-1}\left(\left[c_{1}, c_{3}\right]\right) \backslash$ Sing $Y^{j}$ are isolated and non-degenerate for all $j$, and that $\tilde{\varphi}$ is $n$-convex on a neighborhood of $Y^{0}$

Let $\Sigma$ be the union of the sets of critical points of $\tilde{\varphi} \mid\left(Y \cap \varphi^{-1}\left(\left[c_{1}, c_{3}\right]\right) \backslash\right.$ Sing $\left.Y^{j}\right)$ for all $j>0$.

Since $\tilde{\varphi} \mid\left(Y^{j} \cap \varphi^{-1}\left(\left[c_{1}, c_{3}\right]\right) \backslash \operatorname{Sing} Y^{j}\right)$ have no local maximums, one can find an arbitrarily small neighborhood $U$ of $\Sigma$ and a $C^{\infty}$ diffeomorphism $F_{v}$ : $Y \longrightarrow Y$ fixing the points of $\Sigma$ and $Y \backslash U$, such that $\tilde{\varphi} \circ F_{v}$ is $n$-convex on a neighborhood of $\Sigma$. As such a diffeomorphism $F_{v}$, it suffices to take one with sufficiently enlarging dilation along a positive direction, compared to the complementary directions, of the Hessian of $\tilde{\varphi} \mid\left(Y^{j} \backslash \operatorname{Sing} Y^{j}\right)$ at $\Sigma$.

Then it is obvious that one can find a $C^{\infty}$ convex increasing function $\lambda$ on $\mathbb{R}$ such that $\lambda \circ \tilde{\varphi} \circ F_{v}$ satisfies the requirements for $\Phi$ for some $\varepsilon$ and $C$.

The following is contained in [O-3]. Although the notations are slightly different from that of [O-3], the adjustment is routine and may well be left to the reader.

Lemma 2 (See [O-3, Proposition (in §2) and §3]). Let $X$ be a complex analytic space whose irreducible components are noncompact, and let $\xi: X \longrightarrow \mathbb{R}$ be a $C^{\infty}$ exhaustion function such that $\xi$ is n-convex on a neighborhood of Sing $X$. Then there exists a convex increasing function $\lambda$ on $\mathbb{R}$ and a $C^{\infty} n$ convex exhaustion function $\Psi$ on $X$ such that $\Psi=\lambda \circ \xi$ holds on a neighborhood of SingX. 
Therefore, by a construction inductive on the dimension, one can find a $C^{\infty}$ n-convex exhaustion function $\varphi$ on $X \backslash|D|$ such that $\varphi \mid\left(X^{j} \backslash|D|\right)$ have no local maximums outside a compact subset, for any $\mathrm{j}>0$.

Proof of Theorem 1. Let $\eta: X \longrightarrow \mathbb{R}$ be any $C^{\infty}$ function which is n-convex on a neighborhood of $|D|$. Existence of such a function $\eta$ is obvious. Replacing $\eta$ by $B \eta$ for a sufficiently large constant $B$, if necessary, we may assume that there exists a compact set $K_{1} \subset X \backslash|D|$ such that $\psi:=-\log |s|^{2}+\eta$ is $n$-convex on $X \backslash|D| \backslash K_{1}$.

Clearly, we may choose $K_{1}$ in such a way that $\psi \mid\left(X^{j} \backslash|D| \backslash K_{1}\right)$ have no local maximums if $j>0$.

Hence, in view of the existence of $\varphi$ as above and Lemma 1, there exists a compact set $K_{2} \subset X \backslash|D|$ such that one can extend $\psi \mid\left(X \backslash|D| \backslash K_{2}\right)$ to a $C^{\infty} n$ convex function on $X \backslash|D|$. This was what we wanted to show, as was mentioned before stating Lemma 1.

\section{$\S 3 . \quad$ Proof of Theorem 2}

If $n>\operatorname{dim} f^{-1}(z)$, then for any coherent analytic sheaf $\mathcal{F}$ over $M$ one has $\left(R^{n} f_{*} \mathcal{F}\right)_{z}=0$ by [A-G], since $f^{-1}(z)$ admits an $n$-complete neighborhood system (cf. [B]). If $z$ is as in the assumption and $n=\operatorname{dim} f^{-1}(z)$, then $[D] \mid f^{-1}(z)$ is $n$-concave by Theorem 1 . Hence $[D]$ is $n$-concave on a neighborhood of $f^{-1}(z)$.

Therefore, since $f^{-1}(z)$ admits a holomorphically convex neighborhood system, the result follows from a vanishing theorem of Serre type on weakly 1-complete manifolds (cf. [O-1, Corollary 1.4]).

\section{$\S 4 . \quad$ Proof of Theorem 3}

Similarly as above, it suffices to show the assertion when $n=\operatorname{dim} f^{-1}(z)$. Let $s$ be a canonical section of $[D]$ and let $g$ be any Kähler metric on $M$. By the assumption on $z$, one can find a fiber metric $\mathrm{h}$ of $[D]$ and a holomorphically convex neighbourhood $W$ of $f^{-1}(z)$ in such a way that $([D], h)$ is $n$-concave on $W$. Then, since the function $\eta$ in the proof of Theorem 1 can be chosen in such a way that all the eigenvalues of $\partial \bar{\partial} \eta$ with respect to any prescribed metric are greater than -1 and at most $n-1$ of them are less than $n$, it is easy to see that, in the present situation, one can choose $h$ so that there exists a $C^{\infty}$ (weakly) convex increasing function $\lambda$ satisfying $\lambda^{\prime}(\mathrm{t})=1$ for sufficiently large $t$, such that the eigenvalues of the curvature form of 


$$
\hat{h}:=h|s|^{-2} \exp \left(-\lambda\left(-\log |s|^{2}\right)\right)
$$

with respect to $g$, say $\gamma_{1} \leq \gamma_{2} \leq \cdots \leq \gamma_{N}$, satisfy $\gamma_{1}+\cdots+\gamma_{n} \geq 0$ everywhere and $\gamma_{1}+\cdots+\gamma_{n}>1$ on some neighborhood of $f^{-1}(z) \cap|D|$.

Let $\varphi$ be any $C^{\infty}$ plurisubharmonic function on $W$ such that $g+\partial \bar{\partial} \varphi$ is a complete Kähler metric on $W$. Then there exists a positive number $L$, independent of $\varphi$, such that the sums of $n$ eigenvalues of the curvature form of $\hat{h} \exp (-L \varphi)$ with respect to $g+\partial \bar{\partial} \varphi$ are nonnegative on $W$ and greater than 1 on some neighborhood of $f^{-1}(z) \cap|D|$.

In this situation, it is clear from Nakano's inequality that there exist no nonzero square integrable $K_{M} \otimes[D]$-valued harmonic $(0, n)$-forms on $W$ with respect to the metrics $g+\partial \bar{\partial} \varphi$ and $\hat{h} \exp (-L \varphi)$.

Thus, the vanishing of the $L^{2}$ harmonic forms holds with respect to $(g+$ $\left.\partial \bar{\partial} \varphi^{2}, \hat{h} \exp \left(-L \varphi^{2}\right)\right)$, for any $C^{\infty}$ nonnegative plurisubharmonic exhaustion function $\varphi$ on $W$.

Recall that the analytic sheaf cohomology groups of holomorphically convex manifolds are Hausdorff spaces by the direct image theorem of Grauert (cf. $[\mathrm{G}-2]$ and $[\mathrm{F}-\mathrm{K}])$.

Thus we may conclude that, from the vanishing of the $L^{2}$ harmonic forms, that $\left(R^{n} f_{*} \mathcal{O}\left(K_{M} \otimes[D]\right)\right)_{z}=0$.

\section{Acknowledgment}

The authors are grateful to the referee for pointing out silly mistakes in the manuscript and for asking them to make the proofs of Lemma 1 and Theorem 3 more readable.

\section{References}

[A-G] A. Andreotti and H. Grauert, Théorème de finitude pour la cohomologie des espaces complexes, Bull. Soc. Math. France 90 (1962), 193-259.

[B] D. Barlet, Base de voisinages $n$-complets pour un sous-ensemble analytique compact de dimension $n$. Applications, C. R. Acad. Sci. Paris Sér. A-B 286 (1978), no. 17, A751-A753.

[Dm] J.-P. Demailly, Cohomology of $q$-convex spaces in top degrees, Math. Z. 204 (1990), no. 2, 283-295.

[F-K] O. Forster and K. Knorr, Ein Beweis des Grauertschen Bildgarbensatzes nach Ideen von B. Malgrange, Manuscripta Math. 5 (1971), 19-44.

[F] H. Fuse, Positivity of line bundles associated to point divisors and its parameter dependence, Master Thesis (Japanese), Nagoya Univ., 2009.

[G-1] H. Grauert, Charackterisierung der Holomorphiegebiete durch die vollständige Kählersche Metrik, Math. Ann. 131 (1956), 38-75. 
[G-2] H. Grauert, Selected papers. Vol. I, II, (German) With commentary by Y. T. Siu et al., Springer, Berlin, 1994, Vol. I: xii+439 pp., Vol. II: pp. i-xii and 441-923.

[O-1] T. Ohsawa, Isomorphism theorems for cohomology groups of weakly 1-complete manifolds, Publ. Res. Inst. Math. Sci. 18 (1982), no. 1, 191-232.

[O-2] , Vanishing theorems on complete Kähler manifolds, Publ. Res. Inst. Math. Sci. 20 (1984), no. 1, 21-38.

[O-3] Completeness of noncompact analytic spaces, Publ. Res. Inst. Math. Sci. 20 (1984), no. 3, 683-692.

[S] Y. Siu, Analytic sheaf cohomology groups of dimension $n$ of $n$-dimensional complex spaces, Trans. Amer. Math. Soc. 143 (1969), 77-94. 